DESY 07-155

arXiv:0709.3899 [hep-ph]

SFB/CPP-07-53

LTH 758

September 2007

\title{
On Third-Order Timelike Splitting Functions and Top-Mediated Higgs Decay into Hadrons
}

\author{
S. $\operatorname{Moch}^{a}$ and A. Vogt ${ }^{b}$ \\ ${ }^{a}$ Deutsches Elektronensynchrotron DESY \\ Platanenallee 6, D-15738 Zeuthen, Germany \\ ${ }^{b}$ Department of Mathematical Sciences, University of Liverpool \\ Liverpool L69 3BX, United Kingdom
}

\begin{abstract}
We employ relations between spacelike and timelike deep-inelastic processes in perturbative QCD to calculate the next-to-next-to-leading order (NNLO) contributions to the timelike quark-quark and gluon-gluon splitting functions for the evolution of flavour-singlet fragmentation distributions. We briefly address the end-point behaviour and the numerical size of these third-order corrections, and write down the second moments of all four timelike splitting functions. In the same manner we re-derive the NNLO result for the Higgs-boson decay rate into hadrons in the limit of a heavy top quark and five massless flavours, and confirm the recent $\mathrm{N}^{3} \mathrm{LO}$ computation of this quantity.
\end{abstract}


In this letter we present new results on the scale dependence (evolution) of the parton fragmentation distributions $D_{f}^{h}\left(x, Q^{2}\right)$. Here $x$ denotes the fraction of the momentum of the final-state parton $f$ carried by the outgoing hadron $h$, and $Q^{2}$ is a timelike hard scale, such as the squared fourmomentum of the gauge boson in $e^{+} e^{-} \rightarrow \gamma, Z \rightarrow h+X$. This scale dependence is given by

$$
\frac{d}{d \ln Q^{2}} D_{i}^{h}\left(x, Q^{2}\right)=\int_{x}^{1} \frac{d z}{z} P_{j i}^{T}\left(z, \alpha_{\mathrm{s}}\left(Q^{2}\right)\right) D_{j}^{h}\left(\frac{x}{z}, Q^{2}\right)
$$

where the summation over $j=q, \bar{q}, g$ is understood. The timelike splitting functions $P_{j i}^{T}$ admit an expansion in powers of the strong coupling $\alpha_{s}$,

$$
P_{j i}^{T}\left(x, \alpha_{\mathrm{s}}\left(Q^{2}\right)\right)=a_{\mathrm{s}} P_{j i}^{(0) T}(x)+a_{\mathrm{s}}^{2} P_{j i}^{(1) T}(x)+a_{\mathrm{s}}^{3} P_{j i}^{(2) T}(x)+\ldots,
$$

where we normalize the expansion parameter as $a_{\mathrm{s}} \equiv \alpha_{\mathrm{s}}\left(Q^{2}\right) /(4 \pi)$. The leading-order (LO) terms in Eq. (2) are identical to the spacelike case of the initial-state parton distributions, a fact often referred to as the Gribov-Lipatov relation [1]. Also the next-to-leading order (NLO) contributions $P_{j i}^{(1) T}(x)$ have been known for more than 25 years. These quantities are related to their spacelike counterparts by a suitable analytic continuation [2-4], see also Ref. [5].

In a previous publication [6] we have calculated the next-to-next-to-leading order (NNLO) splitting functions $P_{\mathrm{ns}}^{(2) T}(x)$ for the non-singlet combinations of quark fragmentation distributions. This calculation was based on an analytic continuation of the corresponding unrenormalized partonic structure function in deep-inelastic scattering (DIS) [7], performed after subtracting contributions due to the quark form factor [8] and backed up by another relation between the spacelike and timelike cases conjectured in Ref. [9]. We now address the flavour-singlet timelike evolution

$$
\frac{d}{d \ln Q^{2}}\left(\begin{array}{c}
D_{S} \\
D_{g}
\end{array}\right)=\left(\begin{array}{cc}
P_{\mathrm{qq}}^{T} & P_{\mathrm{gq}}^{T} \\
P_{\mathrm{qg}}^{T} & P_{\mathrm{gg}}^{T}
\end{array}\right) \otimes\left(\begin{array}{c}
D_{S} \\
D_{g}
\end{array}\right) \quad \text { with } \quad D_{S} \equiv \sum_{r=1}^{n_{f}}\left(D_{q_{r}}+D_{\bar{q}_{r}}\right) .
$$

Here $\otimes$ abbreviates the Mellin convolution written out in Eq. (1), and $n_{f}$ stands for the number of effectively massless quark flavours. Specifically, we extend the approach of Ref. [6] to derive the NNLO diagonal entries $P_{\mathrm{qq}}^{(2) T}(x)$ and $P_{\mathrm{gg}}^{(2) T}(x)$ in Eq. (3). The relation of this calculation to Higgs-boson decay will be addressed below.

For brevity focusing on the gluonic case, our calculation starts form the unrenormalized structure function $F_{\phi, g}^{\mathrm{b}}$ for DIS by the exchange of a scalar $\phi$ coupling (like the Higgs-boson in the heavy-top limit) directly only to gluons via $\phi G_{\mu \nu}^{a} G_{a}^{\mu \nu}$, where $G_{\mu \nu}^{a}$ denotes the gluon field strength tensor. This quantity has been computed to three loops for the determination of the spacelike NNLO quark-gluon and gluon-gluon splitting functions in Ref. [10]. In dimensional regularization with $D=4-2 \varepsilon$ its perturbative expansion in terms of the bare reduced coupling $a_{\mathrm{s}}^{\mathrm{b}}$ can be written as

$$
F_{\phi, g}^{\mathrm{b}}\left(a_{\mathrm{s}}^{\mathrm{b}}, Q^{2}\right)=\delta(1-x)+\sum_{l=1}^{\infty}\left(a_{\mathrm{s}}^{\mathrm{b}}\right)^{n}\left(\frac{Q^{2}}{\mu^{2}}\right)^{-n \varepsilon} F_{\phi, g}^{\mathrm{b}(n)} .
$$

The $n$-th order terms $F_{\phi, g}^{\mathrm{b}(n)}$ are then iteratively decomposed into contributions arising from the analogous expansion coefficients $\mathcal{F}_{m \leq n}$ of the $\phi g g$ form factor [11] and remaining 'real' parts $\mathcal{R}_{n}$, 


$$
\begin{aligned}
F_{\phi, g}^{\mathrm{b}(1)} & =2 \mathcal{F}_{1} \delta(1-x)+\mathcal{R}_{1} \\
F_{\phi, g}^{\mathrm{b}(2)} & =2 \mathcal{F}_{2} \delta(1-x)+\left(\mathcal{F}_{1}\right)^{2} \delta(1-x)+2 \mathcal{F}_{1} \mathcal{R}_{1}+\mathcal{R}_{2} \\
F_{\phi, g}^{\mathrm{b}(3)} & =2 \mathcal{F}_{3} \delta(1-x)+2 \mathcal{F}_{1} \mathcal{F}_{2} \delta(1-x)+\left(2 \mathcal{F}_{2}+\left(\mathcal{F}_{1}\right)^{2}\right) \mathcal{R}_{1}+2 \mathcal{F}_{1} \mathcal{R}_{2}+\mathcal{R}_{3} .
\end{aligned}
$$

Note that the functions $\mathcal{R}_{m \geq 2}$ do not only collect tree-level amplitudes but also combinations of real-emission and virtual corrections. As in Ref. [6], this will lead to a problem in the thirdorder analytic continuation. For $P_{\mathrm{gg}}^{(2) T}$, however, this problem can be fixed afterwards in complete analogy to the previous non-singlet quark case (see below), thus we can ignore it for the moment.

The analytic continuation of the form factor to the time-like case is known. The $x$-dependent functions $\mathcal{R}_{n}$ are continued from $x$ to $1 / x$ [2-5], taking into account the (complex) continuation of $q^{2}$ (see Eq. (4.1) of Ref. [8]) and the additional prefactor $x^{1-2 \varepsilon}$ originating from the phase space of the detected parton in the timelike case [12]. We have performed this continuation using routines for the harmonic polylogarithms (HPLs) [13] implemented in FORM [14]. The only subtle point in the analytic continuations is the treatment of logarithmic singularities for $x \rightarrow 1$ starting with

$$
\ln (1-x) \rightarrow \ln (1-x)-\ln x+i \pi
$$

After these analytic continuations the one-parton inclusive fragmentation function in $\phi$-decay is re-assembled order by order analogous to Eq. (5), keeping the real parts of the continued $\mathcal{R}_{n}$ only. Then the renormalization of the operator $G_{\mu \nu}^{a} G_{a}^{\mu \nu}$ and the strong coupling constant is performed. Finally the timelike splitting functions (and coefficient functions) can extracted iteratively from the mass factorization relations

$$
\begin{aligned}
F_{\phi, g}^{(1) T}= & -\frac{1}{\varepsilon} P_{\mathrm{gg}}^{(0)}+c_{\phi, \mathrm{g}}^{(1) T}+\varepsilon a_{\phi, \mathrm{g}}^{(1) T}+\varepsilon^{2} b_{\phi, \mathrm{g}}^{(1) T}+\ldots \\
F_{\phi, g}^{(2) T}= & \frac{1}{2 \varepsilon^{2}}\left\{\left(P_{\mathrm{gi}}^{(0)}+\beta_{0} \delta_{\mathrm{gi}}\right) P_{\mathrm{ig}}^{(0)}\right\}-\frac{1}{2 \varepsilon}\left\{P_{\mathrm{gg}}^{(1) T}+2 P_{\mathrm{gi}}^{(0)} c_{\phi, \mathrm{i}}^{(1) T}\right\}+c_{\phi, \mathrm{g}}^{(2) T}-P_{\mathrm{gi}}^{(0)} a_{\phi, \mathrm{i}}^{(1) T} \\
& +\varepsilon\left\{a_{\phi, \mathrm{g}}^{(2) T}-P_{\mathrm{gi}}^{(0)} b_{\phi, \mathrm{i}}^{(1) T}\right\}+\ldots \\
F_{\phi, g}^{(3) T}= & -\frac{1}{6 \varepsilon^{3}}\left\{P_{\mathrm{gi}}^{(0)} P_{\mathrm{ij}}^{(0)} P_{\mathrm{jg}}^{(0)}+3 \beta_{0} P_{\mathrm{gi}}^{(0)} P_{\mathrm{ig}}^{(0)}+2 \beta_{0}^{2} P_{\mathrm{gg}}^{(0)}\right\} \\
& +\frac{1}{6 \varepsilon^{2}}\left\{2 P_{\mathrm{gi}}^{(0)} P_{\mathrm{ig}}^{(1) T}+P_{\mathrm{gi}}^{(1) T} P_{\mathrm{ig}}^{(0)}+2 \beta_{0} P_{\mathrm{gg}}^{(1) T}+2 \beta_{1} P_{\mathrm{gg}}^{(0)}+3 P_{\mathrm{gi}}^{(0)}\left(P_{\mathrm{ij}}^{(0)}+\beta_{0} \delta_{\mathrm{ij}}\right) c_{\phi, \mathrm{j}}^{(1) T}\right\} \\
& -\frac{1}{6 \varepsilon}\left\{2 P_{\mathrm{gg}}^{(2) T}+3 P_{\mathrm{gi}}^{(1) T} c_{\phi, \mathrm{i}}^{(1) T}+6 P_{\mathrm{gi}}^{(0)} c_{\phi, \mathrm{i}}^{(2) T}-3 P_{\mathrm{gi}}^{(0)}\left(P_{\mathrm{ij}}^{(0)}+\beta_{0} \delta_{\mathrm{ij}}\right) a_{\phi, \mathrm{j}}^{(1) T}\right\} \\
& +c_{\phi, \mathrm{g}}^{(3) T}-\frac{1}{2} P_{\mathrm{gi}}^{(1) T} a_{\phi, \mathrm{i}}^{(1) T}-P_{\mathrm{gi}}^{(0)} a_{\phi, \mathrm{i}}^{(2) T}+\frac{1}{2} P_{\mathrm{gi}}^{(0)}\left(P_{\mathrm{ij}}^{(0)}+\beta_{0} \delta_{\mathrm{ij}}\right) b_{\phi, \mathrm{j}}^{(1) T}+\ldots
\end{aligned}
$$

Here all products of $x$-dependent (generalized) functions are to be read as Mellin convolutions or as products in Mellin- $N$ space, employing routines for harmonic sums and their inverse Mellin transform back to $x$-space $[14,15]$. Obviously the determination of $P_{\mathrm{gg}}^{(2) T}$ from Eq. (8) requires the 'off-diagonal' two-loop coefficient function $c_{\phi, \mathrm{q}}^{(2) T}$. This quantity, $a_{\phi, \mathrm{q}}^{(2) T}$ and the corresponding first-order functions (for all these our normalization differs from the standard convention by a factor of two) can be calculated via a direct analytic continuation of $F_{\phi, q}^{\mathrm{b}(1,2)}$. We have checked this 
fact by comparing the corresponding gluonic results for the photon-exchange case to an explicit two-loop calculation to order $\varepsilon$ [16]. At the third order in $\alpha_{s}$, however, this direct continuation fails to correctly reproduce the $\pi^{2}$ contributions already at order $\varepsilon^{-3}$, thus we cannot derive the off-diagonal timelike NNLO splitting functions in this simple manner.

We are now ready to present our results for the diagonal timelike splitting functions. For completeness we start at NLO where we, of course, reproduce the results of Ref. [3]. Adopting the notations of Ref. [13] for the HPLs, the timelike - spacelike differences can be written as

$$
\begin{aligned}
& \delta P_{\mathrm{ps}}^{(1)}(x) \equiv P_{\mathrm{ps}}^{(1) T}(x)-P_{\mathrm{ps}}^{(1) S}(x)= \\
& \quad 8 C_{F} n_{f}\left(-20 / 9 x^{-1}-3-x+56 / 9 x^{2}-\left(3+7 x+8 / 3 x^{2}\right) \mathrm{H}_{0}+2(1+x) \mathrm{H}_{0,0}\right), \\
& \delta P_{\mathrm{gg}}^{(1)}(x) \equiv P_{\mathrm{gg}}^{(1) T}(x)-P_{\mathrm{gg}}^{(1) S}(x)= \\
& \quad 8 C_{A}^{2}\left(p_{\mathrm{gg}}(x)\left[11 / 3 \mathrm{H}_{0}-4\left(\mathrm{H}_{0,0}+\mathrm{H}_{1,0}+\mathrm{H}_{2}\right)\right]+\left[6(1-x)-22 / 3\left(x^{-1}-x^{2}\right)\right] \mathrm{H}_{0}\right. \\
& \left.\quad-8(1+x) \mathrm{H}_{0,0}\right)-16 / 3 C_{A} n_{f} p_{\mathrm{gg}}(x) \mathrm{H}_{0}+8 C_{F} n_{f}\left(20 / 9 x^{-1}+3+x-56 / 9 x^{2}\right. \\
& \left.\quad+\left[4+6 x+4 / 3\left(x^{-1}+x^{2}\right)\right] \mathrm{H}_{0}+2(1+x) \mathrm{H}_{0,0}\right)
\end{aligned}
$$

where we have used the abbreviation

$$
p_{\mathrm{gg}}(x)=1 /(1-x)+1 / x-2+x-x^{2} .
$$

Note that the non-HPL terms in Eqs. (9) and (10) are identical up to an overall sign, cf. Ref. [9]. The functions $P_{\mathrm{ps}}^{T, S}$ denote the 'pure singlet' contributions from which the quark-quark entries in, e.g., Eq. (3) are obtained by adding the corresponding non-singlet quantities.

The difference between the timelike NNLO pure-singlet splitting function and its spacelike counterpart of Ref. [10] reads

$$
\begin{aligned}
\delta P_{\mathrm{ps}}^{(2)} & (x) \equiv P_{\mathrm{ps}}^{(2) T}(x)-P_{\mathrm{ps}}^{(2) S}(x)= \\
+ & 8 C_{A} C_{F} n_{f}\left(269 / 6 x^{-1}+14+113 / 2 x-346 / 3 x^{2}+\zeta_{2}\left(172+167 x+8 x^{2}\right) / 3\right. \\
& -\zeta_{3}\left(12 x^{-1}-13+65 x-28 x^{2}\right)-2(1+x)\left[16 \zeta_{2}^{2}+4 \mathrm{H}_{-1,0,0}+9 \mathrm{H}_{3,0}+4 \mathrm{H}_{3,1}\right. \\
& \left.+10 \zeta_{2} \mathrm{H}_{2}-12 \mathrm{H}_{2,0,0}-2 \mathrm{H}_{2,1,0}-6 \mathrm{H}_{2,2}-\mathrm{H}_{4}\right]+8 / 3\left(x^{-1}+x^{2}\right)\left[4 \mathrm{H}_{-1,0,0}+\zeta_{2} \mathrm{H}_{0}\right] \\
& -2(1-x)\left[8\left(\mathrm{H}_{-3,0}+\mathrm{H}_{-2,0,0}\right)+5 \zeta_{2} \mathrm{H}_{1}-9 \zeta_{2} \mathrm{H}_{0}+25 / 12 \mathrm{H}_{1,0}-6 \mathrm{H}_{1,0,0}-\mathrm{H}_{1,1,0}\right. \\
& \left.-3 \mathrm{H}_{1,2}+\mathrm{H}_{2,1}\right]+8 / 3\left(x^{-1}-x^{2}\right)\left[6 \mathrm{H}_{1,0,0}+\mathrm{H}_{1,1,0}+3 \mathrm{H}_{1,2}-5 \zeta_{2} \mathrm{H}_{1}-\mathrm{H}_{2,0}-\mathrm{H}_{2,1}\right] \\
& +2 / 3\left(4 x^{-1}+27-63 x+28 x^{2}\right) \mathrm{H}_{-2,0}-2 / 3\left(20 x^{-1}-27+9 x+56 x^{2}\right) \mathrm{H}_{-1,0} \\
& +\left(89 / 9 x^{-1}+55+1021 / 6 x+2297 / 18 x^{2}-46 \zeta_{3}-22 x \zeta_{3}\right) \mathrm{H}_{0}-\left(8 / 9 x^{-1}\right. \\
& \left.+293 / 6+370 / 3 x+538 / 9 x^{2}+2 \zeta_{2}(1-7 x)\right) \mathrm{H}_{0,0}-32 x \mathrm{H}_{0,0,0,0}+\left(5-16 / 3 x^{-1}\right. \\
& +85 x) \mathrm{H}_{0,0,0}-1 / 6\left(115 x^{-1}+362-292 x-185 x^{2}\right) \mathrm{H}_{1}-\left(6 x^{-1}+48+59 x\right. \\
& \left.\left.+22 x^{2}\right) \mathrm{H}_{2}-2\left(5+x-8 / 3 x^{2}\right) \mathrm{H}_{2,0}+4\left(2 / 3 x^{-1}+x+2 x^{2}\right) \mathrm{H}_{3}\right)
\end{aligned}
$$




$$
\begin{aligned}
+ & 8 C_{F}^{2} n_{f}\left(-217 / 18-55 / 3 x^{-1}+122 / 9 x+101 / 6 x^{2}+\zeta_{3}\left(16 x^{-1}+36+24 x\right)\right. \\
& -\zeta_{2}\left(127+188 x+128 x^{2}\right) / 3+2(1+x)\left[16 \zeta_{2}^{2}+17 \zeta_{3} \mathrm{H}_{0}+8 \zeta_{2} x \mathrm{H}_{0}-7 \zeta_{2} \mathrm{H}_{0,0}\right. \\
& \left.+10 \zeta_{2} \mathrm{H}_{2}+9 \mathrm{H}_{2,0}-12 \mathrm{H}_{2,0,0}-2 \mathrm{H}_{2,1,0}-6 \mathrm{H}_{2,2}+9 \mathrm{H}_{3,0}+4 \mathrm{H}_{3,1}-\mathrm{H}_{4}\right] \\
& +2(1-x)\left[5 \zeta_{2} \mathrm{H}_{1}+2 \mathrm{H}_{2,0}+139 / 12 \mathrm{H}_{1,0}-6 \mathrm{H}_{1,0,0}-\mathrm{H}_{1,1,0}-3 \mathrm{H}_{1,2}+\mathrm{H}_{2,1}\right] \\
& +8 / 3\left(x^{-1}-x^{2}\right)\left[5 \zeta_{2} \mathrm{H}_{1}+5 / 3 \mathrm{H}_{1,0}-6 \mathrm{H}_{1,0,0}-\mathrm{H}_{1,1,0}-3 \mathrm{H}_{1,2}+2 \mathrm{H}_{2,0}+\mathrm{H}_{2,1}\right] \\
& -\left(527+2473 x+811 x^{2}+72 \zeta_{2}\right) / 18 \mathrm{H}_{0}+\left(62+81 / 2 x+208 / 9 x^{2}\right) \mathrm{H}_{0,0} \\
& +\left(6+18 x-8 x^{2}\right) \mathrm{H}_{0,0,0}+\left(385 / 18 x^{-1}+190 / 3-143 / 3 x-667 / 18 x^{2}\right) \mathrm{H}_{1} \\
& \left.+\left(28 / 9 x^{-1}+71+46 x+248 / 9 x^{2}\right) \mathrm{H}_{2}-4 / 3\left(4 x^{-1}-6-3 x+8 x^{2}\right) \mathrm{H}_{3}\right) \\
+ & 8 C_{F} n_{f}^{2}\left(2 / 9\left(23 x-2 x^{-1}-20-x^{2}\right)+2(1+x)\left[\zeta_{3}-\zeta_{2} \mathrm{H}_{0}-\mathrm{H}_{1}+\mathrm{H}_{2,0}+\mathrm{H}_{3}\right.\right. \\
& \left.-\mathrm{H}_{0,0,0}\right]-(1-x)\left(\mathrm{H}_{1}-\mathrm{H}_{1,0}\right)+4 / 3\left(x^{-1}-x^{2}\right) \mathrm{H}_{1,0}+2 / 9\left(3+18 x+10 x^{2}\right) \mathrm{H}_{0} \\
& \left.-\left(7+x-4 x^{2}\right) / 3 \mathrm{H}_{0,0}-\left(20 x^{-1}-56 x^{2}\right) / 9 \mathrm{H}_{1}+\left(3+7 x+8 / 3 x^{2}\right)\left(\zeta_{2}-\mathrm{H}_{2}\right)\right) .
\end{aligned}
$$

The corresponding result for the gluon-gluon splitting functions is given by

$$
\begin{aligned}
\delta P_{\mathrm{gg}}^{(2)} & (x) \equiv P_{\mathrm{gg}}^{(2) T}(x)-P_{\mathrm{gg}}^{(2) S}(x)= \\
+ & 16 C_{A}^{3}\left(p _ { \mathrm { gg } } ( x ) \left[\left(1025 / 54-11 / 3 \zeta_{2}-2 \zeta_{3}\right) \mathrm{H}_{0}-49 / 3 \mathrm{H}_{0,0}-33 \mathrm{H}_{0,0,0}+16 \mathrm{H}_{0,0,0,0}\right.\right. \\
& -\left(268 / 9-8 \zeta_{2}\right)\left(\mathrm{H}_{1,0}+\mathrm{H}_{2}\right)-44 / 3\left(\mathrm{H}_{1,0,0}+\mathrm{H}_{2,0}+\mathrm{H}_{3}\right)+12 \mathrm{H}_{1,0,0,0}+4 \mathrm{H}_{2,0,0} \\
& \left.+4 \mathrm{H}_{3,0}+12 \mathrm{H}_{4}\right]+p_{\mathrm{gg}}(-x)\left[16 \mathrm{H}_{-3,0}-16 \zeta_{2} \mathrm{H}_{-2}-16 \mathrm{H}_{-2,-1,0}-22 / 3 \mathrm{H}_{-2,0}\right. \\
& +28 \mathrm{H}_{-2,0,0}+8 \mathrm{H}_{-2,2}-16 \mathrm{H}_{-1,-2,0}-32 \mathrm{H}_{-1,-1,0,0}-16 \zeta_{2} \mathrm{H}_{-1,0}-44 / 3 \mathrm{H}_{-1,0,0} \\
& +36 \mathrm{H}_{-1,0,0,0}+8 \mathrm{H}_{-1,2,0}+16 \mathrm{H}_{-1,3}+\left(14 \zeta_{3}-11 / 3 \zeta_{2}\right) \mathrm{H}_{0}+16 \zeta_{2} \mathrm{H}_{0,0}+11 \mathrm{H}_{0,0,0} \\
& \left.-16 \mathrm{H}_{0,0,0,0}-4 \mathrm{H}_{3,0}-12 \mathrm{H}_{4}\right]+(1+x)\left[-24 \mathrm{H}_{-2,0}-48 \mathrm{H}_{-1,0,0}+14 / 3 \mathrm{H}_{2,0}\right. \\
& \left.+28 / 3 \mathrm{H}_{3}\right]+(1-x)\left[32\left(\mathrm{H}_{-3,0}+\mathrm{H}_{-2,0,0}\right)-\left(881 / 36-24 \zeta_{3}\right) \mathrm{H}_{0}-27\left(\mathrm{H}_{1,0}+\mathrm{H}_{2}\right)\right] \\
& -44 / 3\left(x^{-1}+x^{2}\right)\left[2 \mathrm{H}_{-2,0}+4 \mathrm{H}_{-1,0,0}-\mathrm{H}_{2,0}-2 \mathrm{H}_{3}\right]+\left(x^{-1}-x^{2}\right)\left[2261 / 54 \mathrm{H}_{0}\right. \\
& \left.+134 / 9\left(\mathrm{H}_{1,0}+\mathrm{H}_{2}\right)\right]-\left(44 x^{-1}+86+14 x+132 x^{2}\right) / 3 \zeta_{2} \mathrm{H}_{0}+\left(536 x^{-1}+425\right. \\
& \left.\left.+515 x+752 x^{2}+288 \zeta_{2}\right) / 9 \mathrm{H}_{0,0}+\left(88 x^{-1}-10+8 x+44 x^{2}\right) \mathrm{H}_{0,0,0}+64 x \mathrm{H}_{0,0,0,0}\right) \\
+ & 16 C_{A}^{2} n_{f}\left(p _ { \mathrm { gg } } ( x ) \left[-\left(158 / 27-2 / 3 \zeta_{2}\right) \mathrm{H}_{0}-4 / 9 \mathrm{H}_{0,0}+6 \mathrm{H}_{0,0,0}+40 / 9\left(\mathrm{H}_{1,0}+\mathrm{H}_{2}\right)\right.\right. \\
+ & \left.8 / 3\left(\mathrm{H}_{1,0,0}+\mathrm{H}_{2,0}+\mathrm{H}_{3}\right)\right]+2 / 3 p_{\mathrm{gg}}(-x)\left[2 \mathrm{H}_{-2,0}+4 \mathrm{H}_{-1,0,0}+\zeta_{2} \mathrm{H}_{0}-3 \mathrm{H}_{0,0,0}\right] \\
& -\frac{4}{3}(1+x)\left[\zeta_{2} \mathrm{H}_{0}-\mathrm{H}_{2,0}-2 \mathrm{H}_{3}\right]-(1-x)\left[173 / 9 \mathrm{H}_{0}+2\left(\mathrm{H}_{1,0}+\mathrm{H}_{2}\right)\right]+\left(x^{-1}-x^{2}\right) \\
& {\left.\left[913 / 54 \mathrm{H}_{0}+26 / 9\left(\mathrm{H}_{1,0}+\mathrm{H}_{2}\right)\right]+4 / 9\left(35 x^{-1}+21+48 x\right) \mathrm{H}_{0,0}+4(1+4 x) \mathrm{H}_{0,0,0}\right) } \\
+ & \frac{16}{27} C_{A} n_{f}^{2}\left(p_{\mathrm{gg}}(x)\left[10 \mathrm{H}_{0}+12 \mathrm{H}_{0,0}\right]+12(1+x) \mathrm{H}_{0,0}+\left(13\left(x^{-1}-x^{2}\right)-9+9 x\right) \mathrm{H}_{0}\right)
\end{aligned}
$$




$$
\begin{aligned}
& +8 C_{A} C_{F} n_{f}\left(-2 p_{\mathrm{gg}}(x) \mathrm{H}_{0}-269 / 6 x^{-1}-14-113 / 2 x+346 / 3 x^{2}-\zeta_{2}(172+167 x\right. \\
& \left.+8 x^{2}\right) / 3+\zeta_{3}\left(12 x^{-1}-13+65 x-28 x^{2}\right)+2(1+x)\left[16 \zeta_{2}^{2}+2 \mathrm{H}_{-2,0}-4 \mathrm{H}_{-1,0,0}\right. \\
& +17 \zeta_{3} \mathrm{H}_{0}+4 / 3 \zeta_{2} \mathrm{H}_{0}-3 \zeta_{2} \mathrm{H}_{0,0}+10 \zeta_{2} \mathrm{H}_{2}-12 \mathrm{H}_{2,0,0}-2 \mathrm{H}_{2,1,0}-6 \mathrm{H}_{2,2}+4 \mathrm{H}_{3,1} \\
& \left.+9 \mathrm{H}_{3,0}-\mathrm{H}_{4}\right]+8 / 3\left(x^{-1}+x^{2}\right)\left[4 \mathrm{H}_{-1,0,0}+\zeta_{2} \mathrm{H}_{0}\right]-2(1-x)\left[8\left(\mathrm{H}_{-3,0}+\mathrm{H}_{-2,0,0}\right)\right. \\
& \left.+18 \mathrm{H}_{-2,0}+9 \zeta_{2} \mathrm{H}_{0}+6 \zeta_{3} \mathrm{H}_{0}+4 \zeta_{2} \mathrm{H}_{0,0}-145 / 12 \mathrm{H}_{1,0}\right]+\left[\frac{8}{3}\left(x^{-1}-x^{2}\right)+2(1-x)\right] \\
& {\left[3 \mathrm{H}_{-2,0}-11 / 3 \mathrm{H}_{1,0}+5 \zeta_{2} \mathrm{H}_{1}-6 \mathrm{H}_{1,0,0}-\mathrm{H}_{1,1,0}-3 \mathrm{H}_{1,2}+\mathrm{H}_{2,1}\right]+\left(40 x^{-1}-54\right.} \\
& \left.+18 x+112 x^{2}\right) / 3 \mathrm{H}_{-1,0}-\left(59 x^{-1}+45+1081 / 6 x+157 / 2 x^{2}\right) \mathrm{H}_{0}-\left(464 x^{-1}\right. \\
& \left.+329 / 2-146 x-66 x^{2}\right) / 9 \mathrm{H}_{0,0}-\left(80 / 3 x^{-1}-17+15 x\right) \mathrm{H}_{0,0,0}-32 x \mathrm{H}_{0,0,0,0} \\
& +\left(115 x^{-1}+362-292 x-185 x^{2}\right) / 6 \mathrm{H}_{1}-1 / 9\left(34 x^{-1}-546-417 x-286 x^{2}\right) \mathrm{H}_{2} \\
& \left.+\left(8 x^{-1}+10-14 x-24 x^{2}\right) / 3 \mathrm{H}_{2,0}-8 / 3\left(x^{-1}+5+13 / 2 x+3 x^{2}\right) \mathrm{H}_{3}\right) \\
& +8 C_{F}^{2} n_{f}\left(217 / 18+55 / 3 x^{-1}-122 / 9 x-101 / 6 x^{2}-\zeta_{3}\left(16 x^{-1}+36+24 x\right)+\zeta_{2} / 3\right. \\
& \left(127+188 x+128 x^{2}\right)-2(1+x)\left[16 \zeta_{2}^{2}+\zeta_{2} \mathrm{H}_{0,0}+10 \zeta_{2} \mathrm{H}_{2}+17 \zeta_{3} \mathrm{H}_{0}-12 \mathrm{H}_{2,0,0}\right. \\
& \left.-2 \mathrm{H}_{2,1,0}-6 \mathrm{H}_{2,2}+9 \mathrm{H}_{3,0}+4 \mathrm{H}_{3,1}-\mathrm{H}_{4}\right]-\left[\frac{8}{3}\left(x^{-1}-x^{2}\right)+2(1-x)\right]\left[5 \zeta_{2} \mathrm{H}_{1}\right. \\
& \left.+3 \mathrm{H}_{1,0}-6 \mathrm{H}_{1,0,0}-3 \mathrm{H}_{1,2}-\mathrm{H}_{1,1,0}+\mathrm{H}_{2,1}\right]+\left(4 x^{-1}+283 / 6+239 / 2 x+739 / 18 x^{2}\right. \\
& \left.-8 \zeta_{2}-20 \zeta_{2} x-16 / 3 \zeta_{2} x^{2}\right) \mathrm{H}_{0}-\left(18+97 / 2 x+16 x^{2}\right) \mathrm{H}_{0,0}-\left(6-6 x+8 x^{2}\right) \mathrm{H}_{0,0,0} \\
& -\left(385 x^{-1}+1140-858 x-667 x^{2}\right) / 18 \mathrm{H}_{1}+53 / 6(1-x) \mathrm{H}_{1,0}-\left(20 / 3 x^{-1}+45\right. \\
& \left.\left.+72 x+24 x^{2}\right) \mathrm{H}_{2}-\left(32 / 3 x^{-1}+14+6 x\right) \mathrm{H}_{2,0}-\left(16 / 3 x^{-1}-8-12 x\right) \mathrm{H}_{3}\right) \\
& +8 / 9 C_{F} n_{f}^{2}\left(4 x^{-1}+40-46 x+2 x^{2}-9 \zeta_{2}\left(3+7 x+8 / 3 x^{2}\right)-6(1+x)\left[3 \zeta_{3}+\zeta_{2} \mathrm{H}_{0}\right.\right. \\
& \left.+3 \mathrm{H}_{0,0,0}-\mathrm{H}_{2,0}-5 \mathrm{H}_{3}\right]-\left(92 / 3 x^{-1}-6+48 x-32 / 3 x^{2}\right) \mathrm{H}_{0}-\left(16 x^{-1}+83\right. \\
& \left.+101 x+28 x^{2}\right) \mathrm{H}_{0,0}+\left(20 x^{-1}+27+9 x-56 x^{2}\right) \mathrm{H}_{1}+\left(4 x^{-1}+3-3 x-4 x^{2}\right) \mathrm{H}_{1,0} \\
& \left.+\left(16 x^{-1}+39+51 x+8 x^{2}\right) \mathrm{H}_{2}\right) \text {. }
\end{aligned}
$$

Eqs. (11) and (12) represent the main new results of this letter. Analogous to the non-singlet result of Ref. [6], the coefficient of $C_{A}^{3} p_{\text {gg }}(x) H_{0,0} \zeta_{2}$ in Eq. (12) differs from the result of the analytic continuation as specified above. We have determined the correct coefficient via the momentum sum rule for $n_{f}=0$. The complete $C_{A}^{3}, C_{A}^{2} n_{f}$ and $C_{A} n_{f}^{2}$ parts of Eq. (12) have been independently derived by applying the approach of Ref. [9] to the (non-singlet like) non- $C_{F}$ contributions to $P_{\mathrm{gg}}$. Furthermore an inspection of the above results reveals that also $\delta P_{\mathrm{gg}}^{(2)}+\delta P_{\mathrm{ps}}^{(2)}$ does not receive any non-HPL contributions. Since the determination of these two functions uses largely independent information, we view this fact as another non-trivial check of our calculations.

As predicted in Ref. [9], the large- $x$ behaviour of $P_{\mathrm{gg}}^{(2) T}$ is identical to that of its spacelike counterpart (see Eq. (4.16) of Ref. [10]) up to the sign of the subleading $\ln (1-x)$ contribution. 
The pure-singlet splitting functions are suppressed by two powers of $(1-x)$ for $x \rightarrow 1$. We now turn to the small- $x$ limits. Up to terms suppressed by powers of $x$ the NLO quantities read

$$
\begin{aligned}
& x P_{\mathrm{qq}}^{(1) T}(x)=-\frac{80}{9} C_{F} n_{f}, \\
& x P_{\mathrm{gg}}^{(1) T}(x)=-16 C_{A}^{2} L_{0}^{2}-\frac{8}{3}\left[11 C_{A}^{2}+2 n_{f}\left(C_{A}-2 C_{F}\right)\right] L_{0}-\frac{92}{9} n_{f}\left(C_{A}-2 C_{F}\right)
\end{aligned}
$$

with $L_{0} \equiv \ln x$. The log-enhanced contributions of our new diagonal NNLO splitting functions are

$$
\begin{aligned}
x P_{\mathrm{qq}}^{(2) T}(x)= & -\frac{32}{9} C_{A} C_{F} n_{f}\left(2 L_{0}^{3}+L_{0}^{2}\right)+\frac{8}{27}\left(155+72 \zeta_{2}\right) C_{A} C_{F} n_{f} L_{0}+o(1), \\
x P_{\mathrm{gg}}^{(2) T}(x)= & \frac{64}{3} C_{A}^{3} L_{0}^{4}+\frac{32}{9}\left(33 C_{A}^{3}+6 C_{A}^{2} n_{f}-10 C_{A} C_{F} n_{f}\right) L_{0}^{3} \\
& +\frac{8}{9}\left[\left(389-144 \zeta_{2}\right) C_{A}^{3}+136 C_{A}^{2} n_{f}-232 C_{A} C_{F} n_{f}+4 n_{f}^{2}\left(C_{A}-2 C_{F}\right)\right] L_{0}^{2} \\
& +\frac{8}{27}\left[\left(4076-990 \zeta_{2}-972 \zeta_{3}\right) C_{A}^{3}+\left(739-36 \zeta_{2}\right) C_{A}^{2} n_{f}\right. \\
& \left.-\left(1819-144 \zeta_{2}\right) C_{A} C_{F} n_{f}+108 C_{F}^{2} n_{f}+46 n_{f}^{2}\left(C_{A}-2 C_{F}\right)\right] L_{0}+o(1) .
\end{aligned}
$$

Thus, in contrast to the corresponding spacelike quantities, the NNLO timelike singlet splitting functions receive double-logarithmic contributions with very large coefficients, with the leading term of Eq. (15) agreeing with Refs. [17, 18]. Consequently both $x P_{\mathrm{ps}}^{(2) T}$ and $x P_{\mathrm{gg}}^{(2) T}-$ despite a large cancellation between the leading- and subleading logarithms for the latter quantity - show a huge enhancement already at $x \gtrsim 10^{-3}$ as illustrated in Fig. 1, For this and the next figure the harmonic polylogarithms have been evaluated using the FORTRAN package of Ref. [19].

Returning to the region of medium and large values of $x$, Fig. 2 shows the LO, NLO and NNLO approximations to the diagonal entries in Eq. (3) at a scale relevant to gauge-boson and, maybe, Higgs decay. Also here the higher-order corrections are larger than for the spacelike case, in particular for the gluon-gluon splitting. Nevertheless the perturbative expansion appears wellbehaved at least for $x \gtrsim 0.1$. As usual, the region of safe applicability of the NNLO approximation for Eq. (1) will be wider as an effect of the Mellin convolution.

As mentioned above, we are presently not in a position to derive the $\zeta_{2}$-terms of the offdiagonal quantities $P_{\mathrm{qg}}^{(2) T}(x)$ and $P_{\mathrm{gq}}^{(2) T}(x)$ (with the exception of some $n_{f}$-enhanced contributions). However, their non- $\zeta_{2}$ second moments provide another check, via the momentum sum rule, of our new results (11) and (12). Furthermore, using these results and the momentum sum rule, the missing off-diagonal terms can be reconstructed at $N=2$. Hence we can write down the complete NNLO expressions for the second moments of all four splitting splitting functions,

$$
\begin{aligned}
P_{\mathrm{qq}}^{(2) T}(N=2)=-P_{\mathrm{gq}}^{(2) T}(N=2)=-C_{F}^{3}\left(\frac{54556}{243}-\frac{7264}{27} \zeta_{2}-320 \zeta_{3}+256 \zeta_{2}^{2}\right) \\
-C_{F}^{2} C_{A}\left(\frac{6608}{243}-\frac{2432}{9} \zeta_{2}+\frac{2464}{9} \zeta_{3}-\frac{128}{3} \zeta_{2}^{2}\right)-C_{F} C_{A}^{2}\left(\frac{20920}{243}+\frac{64}{3} \zeta_{3}\right) \\
-C_{F} C_{A} n_{f}\left(\frac{55}{81}+\frac{296}{27} \zeta_{2}-\frac{512}{9} \zeta_{3}\right)-C_{F}^{2} n_{f}\left(\frac{2281}{81}-\frac{32}{9} \zeta_{2}+\frac{64}{9} \zeta_{3}\right),
\end{aligned}
$$



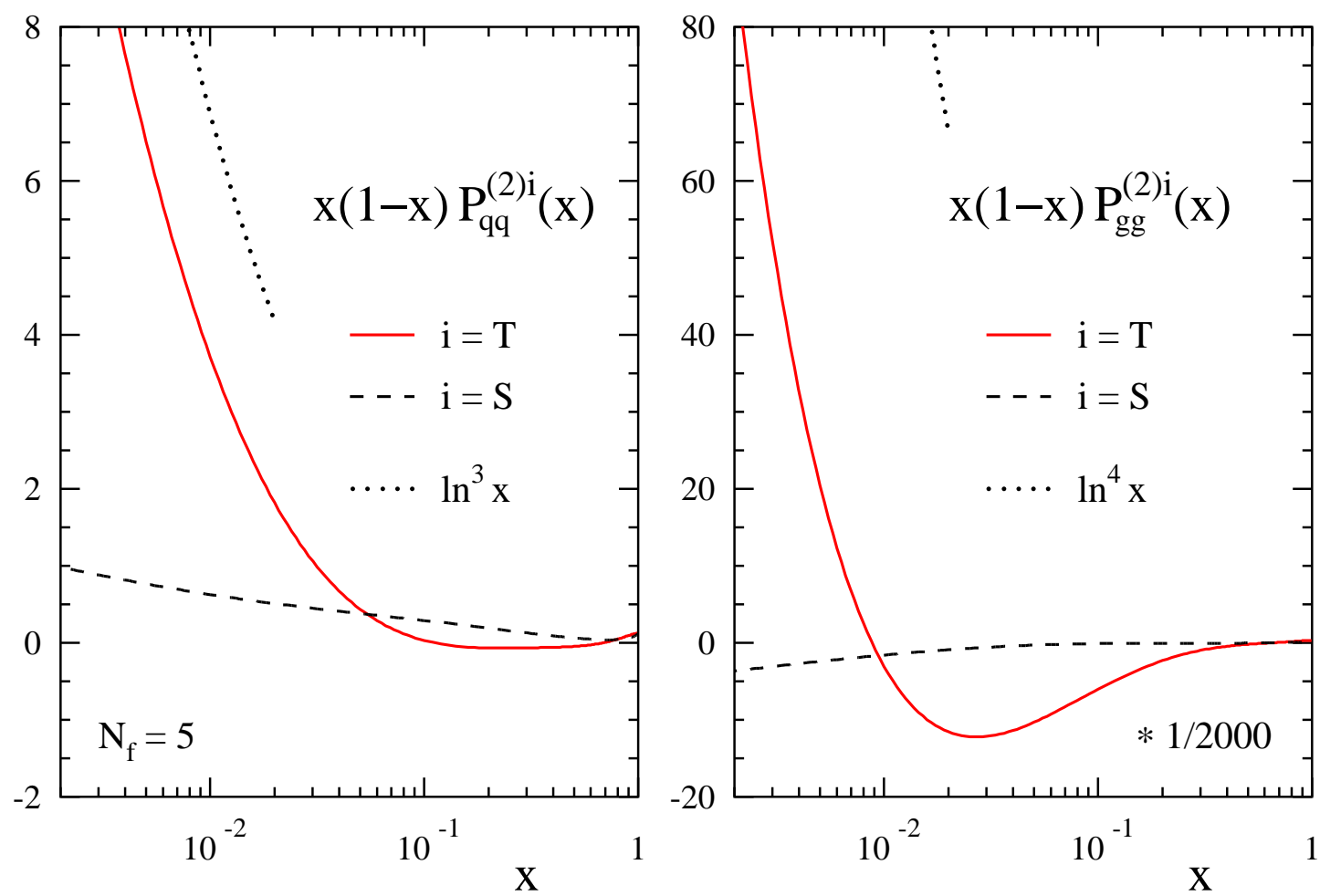

Figure 1: The third-order timelike quark-quark and gluon-gluon splitting functions for five flavours, multiplied by $x(1-x)$ and divided by $2000 \simeq(4 \pi)^{3}$ for display purposes. Also shown are the respective leading small- $x$ contributions and the corresponding spacelike splitting functions.
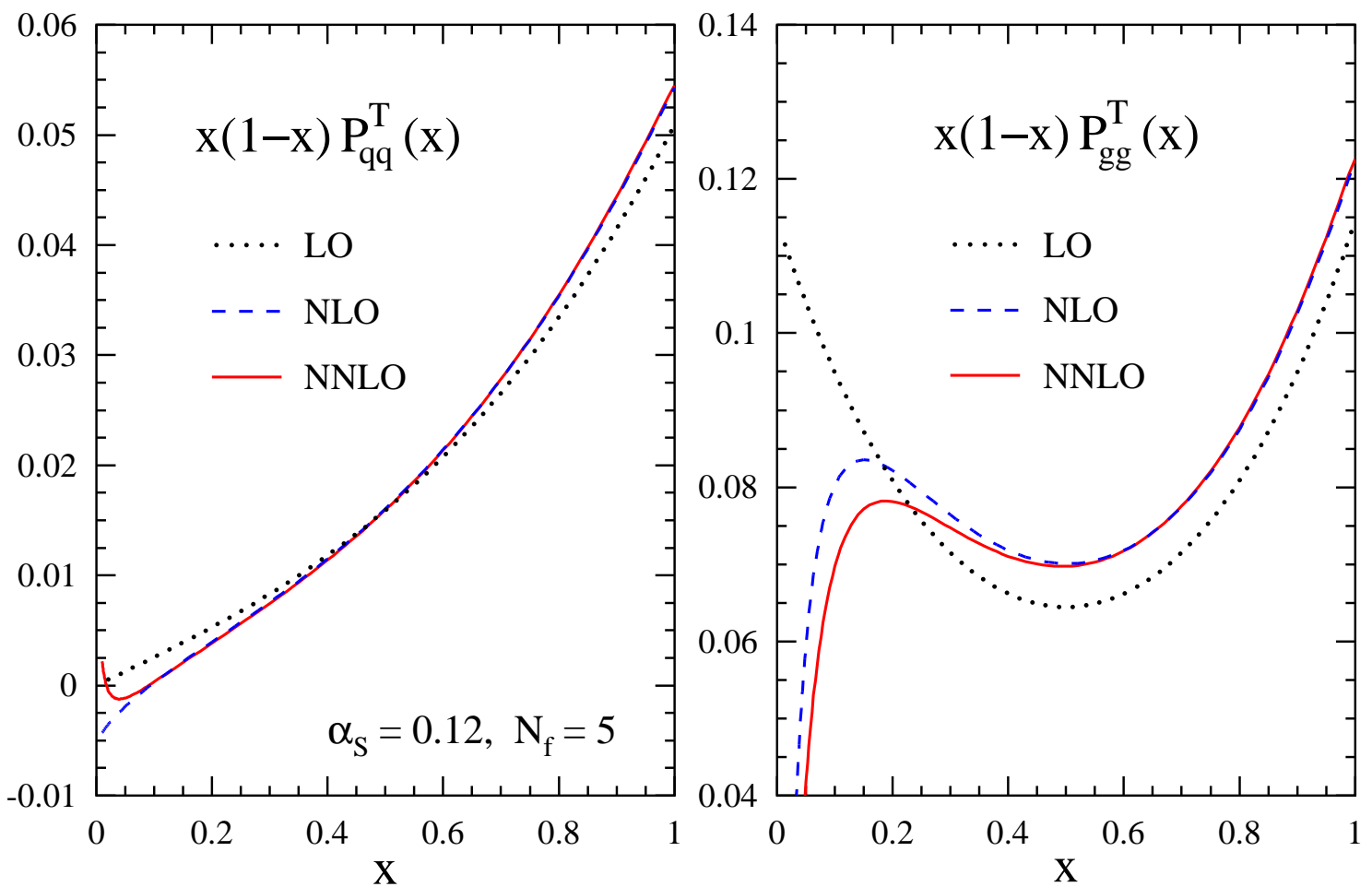

Figure 2: The perturbative expansion of the timelike quark-quark and gluon-gluon splitting functions, again multiplied by $x(1-x)$, at a typical value of the strong coupling constant. 


$$
\begin{aligned}
P_{\mathrm{gg}}^{(2) T}(N & =2)=-P_{\mathrm{qg}}^{(2) T}(N=2)=-C_{A}^{2} n_{f}\left(\frac{6232}{243}-\frac{2132}{27} \zeta_{2}-\frac{128}{9} \zeta_{3}+\frac{160}{3} \zeta_{2}^{2}\right) \\
& +C_{A} n_{f}^{2}\left(\frac{2}{27}-\frac{160}{27} \zeta_{2}+\frac{64}{9} \zeta_{3}\right)-C_{A} C_{F} n_{f}\left(\frac{2681}{243}-\frac{760}{27} \zeta_{2}+\frac{56}{9} \zeta_{3}\right) \\
& -C_{F}^{2} n_{f}\left(\frac{10570}{243}-\frac{352}{27} \zeta_{2}-\frac{32}{9} \zeta_{3}\right)-C_{F} n_{f}^{2}\left(\frac{41}{9}-\frac{128}{27} \zeta_{2}\right) .
\end{aligned}
$$

After inserting the numerical values of the QCD colour factors and the Riemann $\zeta$-function, these results yield, for five light flavours, the benign perturbative expansion

$$
\begin{aligned}
P_{\mathrm{gq}}^{T}\left(N=2, n_{f}=5\right) & \simeq 8 \alpha_{\mathrm{s}} /(9 \pi)\left(1-0.687 \alpha_{\mathrm{s}}+0.447 \alpha_{\mathrm{s}}^{2}+\ldots\right) \\
P_{\mathrm{qg}}^{T}\left(N=2, n_{f}=5\right) & \simeq 5 \alpha_{\mathrm{s}} /(6 \pi)\left(1-1.049 \alpha_{\mathrm{s}}+1.163 \alpha_{\mathrm{s}}^{2}+\ldots\right) .
\end{aligned}
$$

We finally turn to the timelike gluon coefficient functions in Eqs. (6) - (8) and their quark analogues $c_{\phi, \mathrm{q}}^{(n) T}(x)$. In the limit of a heavy top quark and negligible masses of all other flavours, the coefficient functions for the standard-model Higgs-boson differ from those of this scalar $\phi$ only by a perturbative prefactor known to $\mathrm{N}^{3} \mathrm{LO}$ [20]. Thus the latter quantities are directly relevant to one-particle inclusive Higgs decay, and the second-moment combination

$$
\left(C_{\phi, q}^{T}+C_{\phi, g}^{T}\right)(N=2)=1+a_{\mathrm{s}} c_{\phi}^{(1)}+a_{\mathrm{s}}^{2} c_{\phi}^{(2)}+a_{\mathrm{s}}^{3} c_{\phi}^{(3)}+\ldots,
$$

directly enters the Higgs decay rate in the above limit. For the expansion coefficients $c_{\phi}^{(n)}$ our analytic continuations lead to

$$
\begin{aligned}
c_{\phi}^{(1)} & =\frac{73}{3} C_{A}-\frac{14}{3} n_{f}, \\
c_{\phi}^{(2)} & =C_{A}^{2}\left(\frac{37631}{54}-\frac{242}{3} \zeta_{2}-110 \zeta_{3}\right)-C_{A} n_{f}\left(\frac{6665}{27}-\frac{88}{3} \zeta_{2}+4 \zeta_{3}\right) \\
& -C_{F} n_{f}\left(\frac{131}{3}-24 \zeta_{3}\right)+n_{f}^{2}\left(\frac{508}{27}-\frac{8}{3} \zeta_{2}\right), \\
c_{\phi}^{(3)} & =f\left(\zeta_{2}\right)+C_{A}^{3}\left(\frac{15420961}{729}-\frac{178156}{27} \zeta_{3}+\frac{3080}{3} \zeta_{5}\right)+C_{A} n_{f}^{2}\left(\frac{413308}{243}+\frac{56}{9} \zeta_{3}\right) \\
& -C_{A}^{2} n_{f}\left(\frac{2670508}{243}-\frac{9772}{9} \zeta_{3}+\frac{80}{3} \zeta_{5}\right)-C_{F} C_{A} n_{f}\left(\frac{23221}{9}-1364 \zeta_{3}-160 \zeta_{5}\right) \\
& +C_{F}^{2} n_{f}\left(\frac{221}{3}-320 \zeta_{5}+192 \zeta_{3}\right)+C_{F} n_{f}^{2}\left(440-240 \zeta_{3}\right)-n_{f}^{3}\left(\frac{57016}{729}-\frac{64}{27} \zeta_{3}\right)
\end{aligned}
$$

with a function $f\left(\zeta_{2}\right)$ which we cannot derive at this point. Eq. (19) reproduces a well-known NLO result of Refs. [21,22]. Our coefficient (20) represent a completely independent re-derivation of the NNLO expression first obtained in Ref. [23] (see also Ref. [24]). Finally the third-order result (21) provides, despite the missing $\zeta_{2}$-contributions, a highly non-trivial confirmation of the recent $\mathrm{N}^{3} \mathrm{LO}$ calculation of Ref. [25] to which the reader is referred for a further discussion. 
To summarize, we have used relations between spacelike and timelike quantities to compute some important higher-order QCD corrections for timelike processes. We expect that further progress can be achieved along these lines. However this will require improvements on the present decompositions into purely real and real-virtual terms which are beyond the scope of this letter.

FORM and FORTRAN files of our results can be obtained from http://arXiv.org by downloading the source of this article. Furthermore they are available from the authors upon request.

Acknowledgements: This research draws upon (partly still unpublished) results obtained together with J. Vermaseren whom we would like to thank for a very pleasant collaboration. We also thank A. Mitov for stimulating discussions. The work of S.M. has been supported by the Helmholtz Gemeinschaft (contract VH-NG-105) and partly by the Deutsche Forschungsgemeinschaft (SFB/TR 9). During part of this research A.V. enjoyed the hospitality of the Instituut-Lorentz of Leiden University and S.M. that of the Galileo-Galilei Institute in Florence.

\section{References}

[1] V.N. Gribov and L.N. Lipatov, Sov. J. Nucl. Phys. 15 (1972) 438, ibid. 675

[2] G. Curci, W. Furmanski and R. Petronzio, Nucl. Phys. B175 (1980) 27

[3] W. Furmanski and R. Petronzio, Phys. Lett. 97B (1980) 437

[4] E.G. Floratos, C. Kounnas and R. Lacaze, Nucl. Phys. B192 (1981) 417

[5] M. Stratmann and W. Vogelsang, Nucl. Phys. B496 (1997) 41, hep-ph/9612250

[6] A. Mitov, S. Moch and A. Vogt, Phys. Lett. B638 (2006) 61, hep-ph/0604053

[7] S. Moch, J.A.M. Vermaseren and A. Vogt, Nucl. Phys. B688 (2004) 101, hep-ph/0403192

[8] S. Moch, J.A.M. Vermaseren and A. Vogt, JHEP 0508 (2005) 049, hep-ph/0507039

[9] Y.L. Dokshitzer, G. Marchesini and G.P. Salam, Phys. Lett. B634 (2006) 504, hep-ph/0511302

[10] A. Vogt, S. Moch and J.A.M. Vermaseren, Nucl. Phys. B691 (2004) 129, hep-ph/0404111

[11] S. Moch, J. A. M. Vermaseren and A. Vogt, Phys. Lett. B625 (2005) 245, hep-ph/0508055

[12] P.J. Rijken and W.L. van Neerven, Nucl. Phys. B488 (1997) 233, hep-ph/9609377

[13] E. Remiddi and J.A.M. Vermaseren, Int. J. Mod. Phys. A15 (2000) 725, hep-ph/9905237

[14] J.A.M. Vermaseren, math-ph/0010025

[15] J.A.M. Vermaseren, Int. J. Mod. Phys. A14 (1999) 2037, hep-ph/9806280

[16] A. Mitov and S. Moch, Nucl. Phys. B751 (2006) 18, hep-ph/0604160

[17] A.H. Mueller, Phys. Lett. B104 (1981) 161

[18] A. Bassetto, M. Ciafaloni, G. Marchesini and A.H. Mueller, Nucl. Phys. B207 (1982) 189.

[19] T. Gehrmann and E. Remiddi, Comput. Phys. Commun. 141 (2001) 296, hep-ph/0107173

[20] K.G. Chetyrkin, B.A. Kniehl and M. Steinhauser, Nucl. Phys. B510 (1998) 61, hep-ph/9708255

[21] T. Inami, T. Kubota and Y. Okada, Z. Phys. C18 (1983) 69

[22] A. Djouadi, M. Spira and P. M. Zerwas, Phys. Lett. B264 (1991) 440

[23] K.G. Chetyrkin, B.A. Kniehl and M. Steinhauser, Phys. Rev. Lett. 79 (1997) 353, hep-ph/9705240

[24] M. Schreck and M. Steinhauser, arXiv:0708.0916 [hep-ph].

[25] P.A. Baikov and K.G. Chetyrkin, Phys. Rev. Lett. 97 (2006) 061803, hep-ph/0604194 